Peter J. Strouse

Received: 23 April 2004

Accepted: 15 June 2004

Published online: 4 September 2004

(C) Springer-Verlag 2004

P. J. Strouse

Section of Pediatric Radiology,

C.S. Mott Children's Hospital,

University of Michigan Medical Center,

1500 East Medical Center Drive,

Ann Arbor, MI 48109-0252, USA

E-mail: pstrouse@umich.edu

Tel.: + 1-734-7632570

Fax: + 1-734-7649351

\section{Disorders of intestinal rotation and fixation (“malrotation")}

are reviewed and illustrated with particular attention to the child with equivocal imaging findings.

\section{Introduction}

The child with malrotation bears a defect, which, at little notice, may present as an acute life-threatening condition. Occasionally, there are warning signs, but they are non-specific. The clinician must be vigilant. The radiologist must be well versed in the findings of malrotation and volvulus. Malrotation with volvulus is a true surgical emergency (Fig. 1). Prompt diagnosis and treatment may make the difference between life and death for the patient.

\section{Normal embryology}

In order to understand malrotation, a brief review of the embryology of the intestine is required. The anatomical development of the intestinal tract is a complex process. The reader is referred elsewhere for detailed descriptions of this process [1-7]. The rope model, introduced by Snyder and Chaffin [4], nicely allows one to visualize the process (Fig. 2). Frazer and Robbins [2] first described the process of rotation and fixation in terms of three stages. Stage 1 is the period of umbilical cord herniation, lasting from approximately week 5 to week 10. Stage 2 is the period of reduction of the midgut loop back into the abdomen, occurring at weeks 10-11. Stage 3 is the period of fixation, lasting from the end of stage 2 until shortly after birth. Many authors use these three stages of development and distinct $90^{\circ}$ integrals of rotation to describe gut embryology; however, this is somewhat of an oversimplification of a continuous event. Adding to the confusion, the stages are defined slightly differently depending on the author [1-7].

In the early embryo, the gastrointestinal tract is a straight tube. The midgut is supplied by the superior mesenteric artery (SMA), and extends from the bile duct insertion proximally to eventual mid-distal transverse colon distally. The midgut is divided into a cephalad, pre-arterial portion and a caudad, post-arterial portion by the vitelline duct and the SMA. The cephalad midgut contributes the distal duodenum, jejunum and proximal ileum. The caudad midgut contributes the distal ileum, cecum, appendix, and colon extending to the mid-distal transverse colon. 


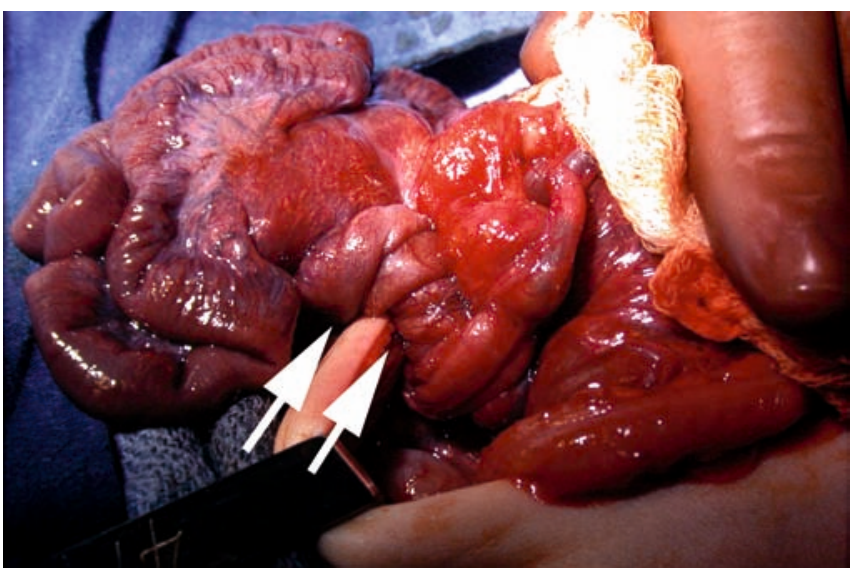

Fig. 1 Intraoperative photograph of an infant with malrotation and volvulus (arrows). The bowel at left is discolored due to ischemia. Courtesy of Arnold G. Coran, M.D., Ann Arbor, MI

The midgut lengthens disproportionately to the embryo. At 6 weeks of gestational age, the midgut herniates in a U-shaped loop into the base of the umbilical cord, entering the extraembryonic coelom. The herniating bowel rotates $90^{\circ}$ around the SMA axis counterclockwise, as viewed facing the embryo from anterior. As a result, the cephalad midgut (the duodenojejunal loop) courses downward right of the SMA and the caudad midgut (the cecocolic loop) courses upward to the left of the SMA. From 6 to 10 weeks gestational age, the midgut remains physiologically herniated. Further
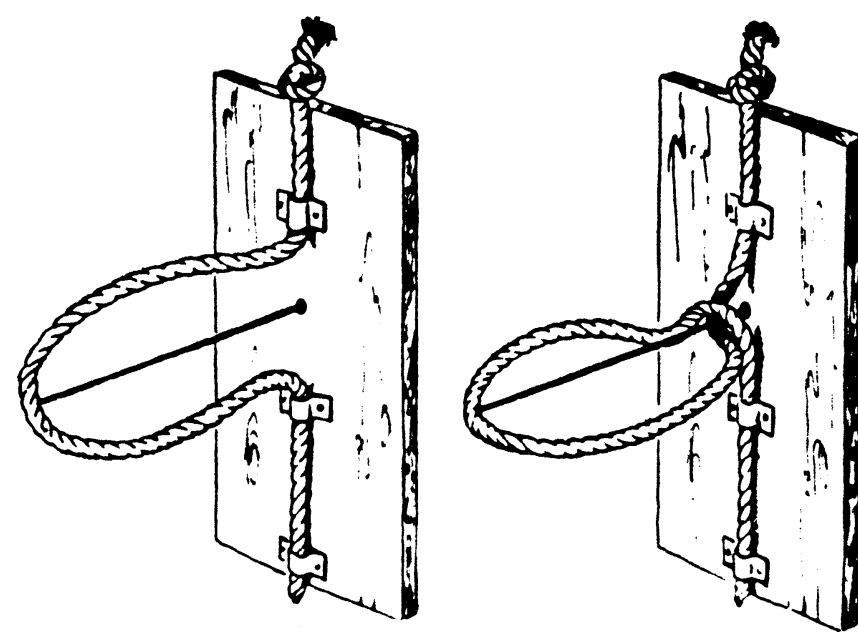

Fig. 2 Rope model of intestinal rotation. The top limb of the loop corresponds to the duodenojejunal loop. The bottom limb of the loop corresponds to the cecocolic loop. The straight wire corresponds to superior mesenteric artery (SMA). At right, the rope loop has been grasped and rotated $270^{\circ}$ or three-quarters of a complete turn in a counterclockwise direction. Note the orientation of the two loops relative to the wire, analogous to rotation of bowel around the SMA. Reproduced with permission from reference 4 elongation of the midgut occurs, predominantly within the cephalad portion. Concomitantly, the duodenojejunal loop undergoes another $90^{\circ}$ counterclockwise rotation. Disproportionate elongation of the proximal small bowel secondarily affects colonic position although the cecocolic loop itself does not undergo further rotation during this period. By 10 weeks, the cecum is well defined and the cecal diverticulum (the appendix) has developed.

At 10 weeks gestation, the bowel re-enters the abdomen. The cephalad midgut (proximal small bowel) enters first, undergoing a third and final $90^{\circ}$ counterclockwise rotation such that the distal duodenum courses inferior and posterior to the SMA and then to the left and upward, completing the normal C-loop duodenal configuration. The caudad midgut (distal ileum, cecum and proximal colon) enters later, undergoing an additional $180^{\circ}$ counterclockwise rotation. As a result, the colon takes its familiar "picture frame" course passing anterior to the SMA with the cecum located to the right. At this stage, the cephalocaudal position of the cecum varies in the right abdomen.

Throughout the remainder of gestation and postnatally for the first few months of life, further elongation of the cecum occurs with descent of the cecum into the right lower quadrant of the abdomen. Fixation of the gut also occurs during this time period. The second, third and fourth portions of duodenum are fixed in the retroperitoneum. The ligament of Treitz, fixating the duodenojejunal junction, is a poorly defined extension from the right diaphragmatic crus and from fibrous tissue around the celiac artery [8]. Descending colon and ascending colon mesenteries fuse with the retroperitoneum fixing these portions of the colon in the retroperitoneum. The transverse mesocolon partially fuses to the greater omentum. The sigmoid mesocolon partially fuses with the retroperitoneum. Both transverse colon and sigmoid colon are left with a small, variable mesenteric attachment. The small bowel is fixed by a broad mesentery, extending from the duodenojejunal junction in the upper left abdomen to the ileocecal valve in the lower right abdomen (Fig. 3a). Although the small bowel is not tightly adherent to the posterior abdominal wall, the broad base of its mesentery stabilizes its position and prevents volvulus.

\section{Abnormal embryology}

Arrest of embryologic development of the midgut can occur at any phase in either or both loops with variable consequences [4]. "Malrotation" is the generic term used to describe the consequences. Simply defined, malrotation is a failure during development of normal rotation of any part of the intestinal tract. For want of a better term, "malrotation" is used similarly in this article. 
Fig. 3 a Normal: the mesenteric root is broad, extending from the duodenojejunal junction in the left upper quadrant to the cecum in the right lower quadrant. b Malrotation: the mesenteric root is narrow, predisposing to volvulus. Reproduced from with permission from: Snyder WH Jr, Chaffin L (1969) Malrotation of the intestine. In: Mustard WT, Ravitch MM, Snyder WH Jr, et al (eds) Pediatric surgery, 2nd edn. Year Book, Chicago, pp 808-817

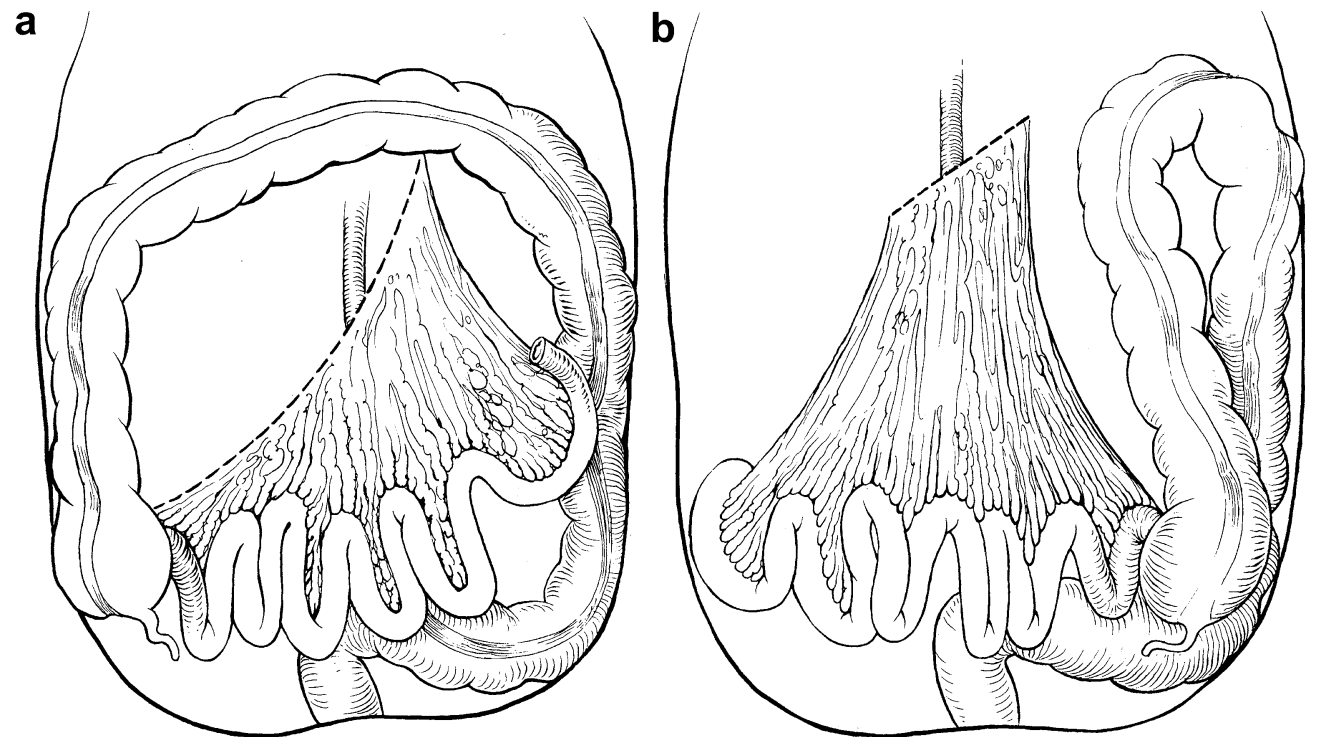

Literally, malrotation means "bad" rotation and thus underplays the importance of malfixation ("bad" fixation) as a predisposing factor to the development of volvulus. Malrotation is not a single distinct entity, but rather a continuum of abnormalities reflecting a failure occurring at any time in the development of the midgut. There are even reports of "hyper-rotation," in which the cecum continues to rotate beyond the normal location, ascending on the left [9].

Schema for categorizing malrotation attempt to define the resultant anomaly based on the presumed timing of the developmental failure $[3,5,6]$. The reader is referred to Stringer and Babyn's [6] text for an excellent discussion of the types of malrotation as to related errors at the different stages of embryologic development. Others have developed classification schema based on the radiographic findings $[10,11]$.

Early failure of rotation yields the pattern of "nonrotation." Nonrotation is a misnomer because the initial $90^{\circ}$ of rotation has occurred with the duodenum lying right of the SMA and the distal colon left of the SMA. Without further rotation, the small bowel is located at the right and the colon is located at the left. Rarely, nonrotation will only affect the duodenum and small bowel with the cecum and colon continuing to undergo normal rotation to assume a normal anatomic location [6]. Long et al. [10] reported a case of nonrotation of the colon with normal duodenal rotation.

"Incomplete rotation" represents a failure occurring during the final $180^{\circ}$ counterclockwise rotation of the small bowel and/or the final $180^{\circ}$ counterclockwise rotation of the colon. Often, this is confusingly termed "malrotation." Other names include "mixed rotation" and "partial rotation" [10]. The resultant abnormality varies from complete non-rotation to normal [6]. Abnormally rotated bowel does not develop a normal mesenteric attachment. The risk for volvulus varies with the degree of mesenteric attachment.

In "reversed rotation" the caudal midgut returns to the abdomen first and the duodenum rotates clockwise, rather than the normal counterclockwise. As a result, in reversed rotation the duodenum courses anterior to the SMA rather than posterior and the colon courses posterior to the SMA rather than anterior [6]. Rarely, reversed duodenal rotation is accompanied by normal colonic rotation. This may result in an internal hernia [6].

Additional abnormalities may be the result of failure of cecal elongation and failure of small bowel and colonic fixation. An undescended cecum is due to failure of the cecum to elongate [5]. An incompletely fixated ascending colon results in a mobile cecum [12]. Because the colon lengthens and fixation proceeds in the first few months of age, the incidence of a high and/or malfixated cecum diminishes with age. Malfixation of the cecum or sigmoid colon may predispose to the development of cecal or sigmoid volvulus later in life; however, these processes are rare in childhood [12]. Malrotation and malfixation of the cecum may also predispose a child to intussusception (Waugh's syndrome) [13]. In a 1985 study by Brereton et al. [13], 41 of 41 children undergoing surgical treatment for intussusception had an "unfixed" cecum.

Internal hernias often represent a failure of bowel fixation [14]. Right paraduodenal hernias are caused by an extrusion of intestinal loops into a pocket of unfused ascending colon mesentery. Left paraduodenal hernias are caused by an extrusion of intestinal loops into a pocket of unfused descending colon mesentery $[5,6,14$, 15]. Left paraduodenal hernias are more common than right. Internal hernia can occur in a variety of other lesscommon sites, also related to incomplete fusion of the 
colonic mesentery $[5,14,15]$. Internal hernias may be cryptic in presentation or may present suddenly with an acute obstruction and potential compromise of the gut [14]. CT and small bowel follow-through contrast studies may suggest the diagnosis, although findings may be subtle [15].

Malfixated midgut with a short mesenteric root is prone to volvulus (Fig. 3b). Volvulus is defined as a twisting of the intestine causing obstruction. Volvulus is derived from Latin "volvo" meaning "to roll." With volvulus, the midgut is rotated around the axis of the superior mesenteric artery. Twists of $720^{\circ}$ and greater are often reported. Increasing degrees of volvulus will obstruct the bowel lumen, lymphatic drainage, venous drainage, and eventually, arterial supply. Malrotation, in itself, is not a surgical emergency; however, it does indicate a potential underlying predisposition to volvulus. Thus, "semi-elective" surgical management may be pursued. Volvulus, on the other hand, is an acute surgical emergency. With volvulus, obstruction of vascular supply may lead to catastrophic results, including death of the patient.

Peritoneal bands, commonly known as Ladd's bands, form due to disordered embryonic attempts to fixate the malpositioned bowel. The bands course from the cecum and proximal colon to the right upper quadrant retroperitoneum, often entrapping the descending and transverse portions of the duodenum (Fig. 4). The bands may cause a variable degree of obstruction ranging up to complete; however, more often than not, the bands are present with no or mild resultant obstruction. With complete obstruction by bands, patients present in utero or at birth with findings mimicking duodenal atresia. The bands may also distort the course of an incompletely obstructed or non-obstructed duodenum in a Zconfiguration [16].

\section{Presentation}

Malrotation with volvulus has infrequently been diagnosed in utero [17]. In most cases, findings suggestive of bowel obstruction have been noted on prenatal sonography [17]. With increasing resolution of ultrasound images and with the advent of fetal MRI, it should not come as a surprise that more children with malrotation will be diagnosed or suspected based on prenatal findings. There are many reports in the literature of children born with volvulus or its sequelae [17]. Some cases of congenital short gut syndrome may represent the sequelae of in utero volvulus, with presumed in utero resorption of infarcted gut and auto-anastomosis of surviving gut to reestablish patency of the intestine [18].

Malrotation is said to occur in approximately 1 in 500 live births [19]. Although a previously undiagnosed patient with malrotation may theoretically present at any juncture of life with an acute volvulus, approximately $80 \%$ of patients with malrotation will present in the first month of life [19-21]. Of those presenting in the first month of life, most will do so in the first week. The classic presentation of malrotation with volvulus is thus that of a neonate with bilious vomiting. The vomiting is
Fig. 4 Ladd's bands extend from the cecum (a) or ascending colon (b) to the right upper quadrant, passing across and variably obstructing the duodenum. Reproduced with permission from reference [21]
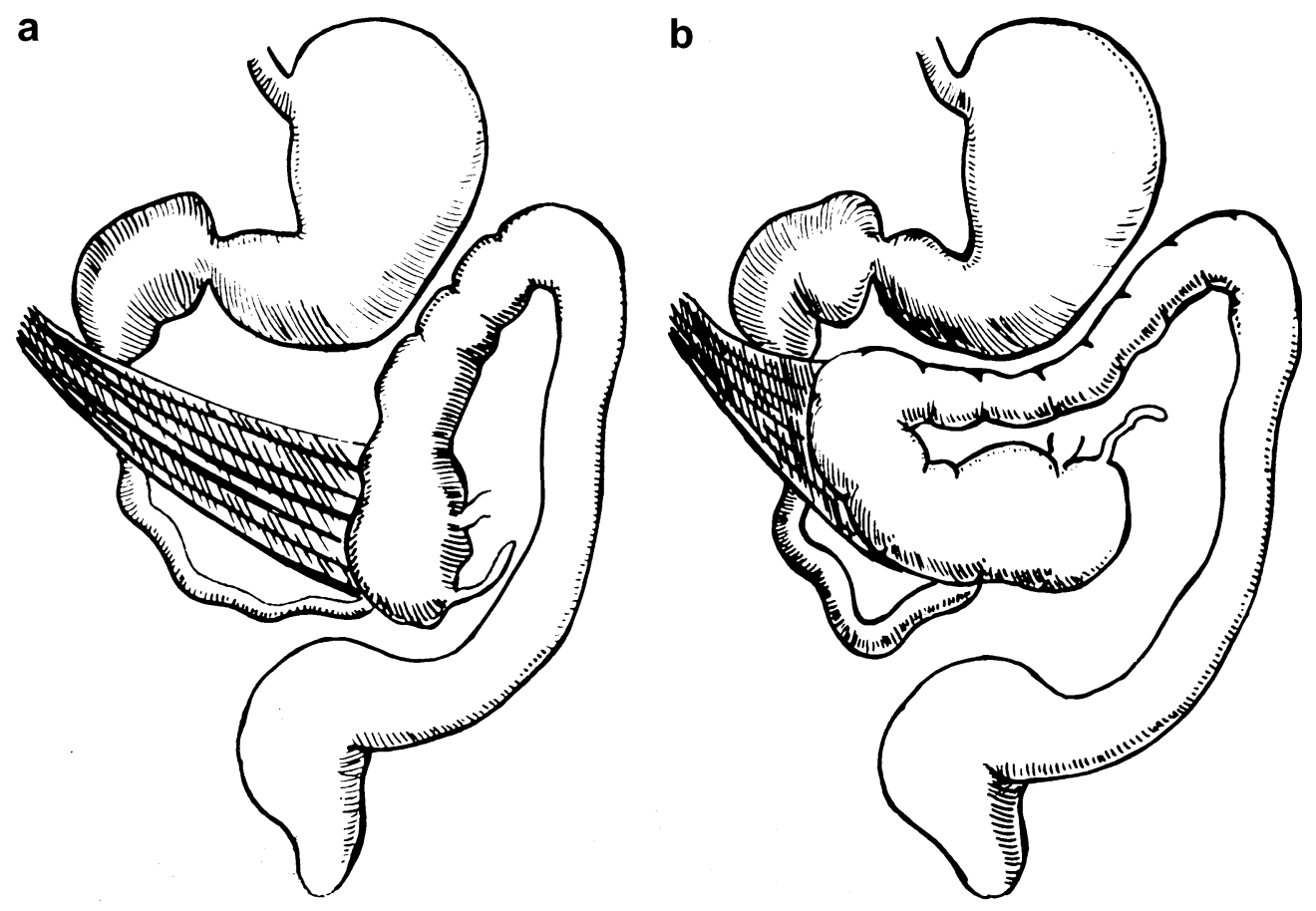
bilious because the point of obstruction is beyond the ampulla of Vater. In contradistinction, the infants with pyloric stenosis have non-bilious vomiting and infants with simple gastroesophageal reflux usually have nonbilious vomiting. Although bilious vomiting should elicit concern for malrotation with volvulus, this symptom is not synonymous with the diagnosis of malrotation [22]. Any obstruction distal to the ampulla of Vater may cause bilious vomiting, particularly in the newborn infant. A majority $(62 \%)$ of infants with bilious vomiting will prove to have no anatomic obstruction, but imaging is necessary to exclude malrotation as an etiology for the vomiting [22].

Apart from vomiting, most patients with malrotation, including many with volvulus, otherwise have a normal history and are without any abnormal physical findings $[19,23]$. Other symptoms may include intermittent abdominal pain, diarrhea and constipation. Hematochezia occurs in $10-15 \%$ of patients with volvulus and portends a poorer prognosis as it is indicative of bowel ischemia [23]. Presentation of malrotation with volvulus as an "acute abdomen" is uncommon [23]. An acute abdomen is not seen until late in the disease process. Patients presenting with shock have a worse prognosis [23]. This manifests as abdominal distension with peritonitis, bloody stools and hemodynamic compromise with hypotension and an elevated heart rate [20]. The signs and symptoms of shock may mislead the clinician and hide the inciting diagnosis of malrotation with volvulus.

Malrotation may also present in an insidious manner with chronic symptoms present or developing over days, months or even years $[24,25]$. In a series by Spigland et al. [24] the mean delay in diagnosis for malrotation presenting beyond the neonatal period was 1.7 years. Patients may have chronic intermittent pain or intermittent vomiting; however, malrotation is present in only a very small fraction of children with either of these symptoms. Older children with malrotation not infrequently have a history of episodes of acute, severe pain. Pain may be accentuated by meals. Patients may be chronically misdiagnosed with other abdominal pain syndromes, "cyclic vomiting," or even psychological disorders. Chronic intermittent volvulus may also interfere with lymphatic and venous drainage from the gut. As a result, the patient may present with malabsorption and/or failure to thrive. A study by Howell et al. [26] noted that $70 \%$ of children presenting with malrotation had clinical evidence of malnutrition. Chylous ascites and mesenteric lymphoceles have been described with chronic lymphatic obstruction due to volvulus [21, 27]. Melena may occur due to chronic mesenteric venous obstruction.

Not infrequently, malrotation is discovered incidentally in a patient undergoing evaluation for other reasons. Some of these patients are truly asymptomatic relative to the malrotation. Others may have had longstanding or intermittent symptoms previously unrecognized or ignored.

\section{Associations}

The majority of children with malrotation do not have any predisposing syndrome or genetic susceptibility. Malrotation is almost invariably present in children with congenital diaphragmatic hernia, gastroschisis and omphalocele $[20,28]$. The incidence of volvulus is rare in these children, probably due to anatomy and due to adhesion of gut occurring after repair of the defect preventing volvulus [28]. Each of these anomalies interferes with normal spatial development of the gut. With congenital diaphragmatic hernia, children with a right defect will have greater malfixation of bowel than children with a left defect [28]. Malrotation has also been reported in association with transient cystic fetal masses, suggesting that a mass-effect from the mass may prevent normal rotation of the bowel [29].

Malrotation is present in the majority of children and adults with heterotaxy syndrome (asplenia/right isomerism and polysplenia/left isomerism) [30-34]. This diagnosis warrants work-up for possible malrotation. The decision as to whether and when to operate on a seemingly asymptomatic infant with heterotaxy syndrome must be balanced with the other medical conditions present. Particularly with asplenia syndrome, the associated congenital heart disease is often severe. The decision to electively operate for malrotation must often be temporized in relation to more pressing demands due to cardiovascular morbidity. Nevertheless, these children may present acutely with volvulus [21, 31, 34]. The incidence of malrotation in children and adults with true situs inversus, as opposed to heterotaxy syndrome, may be slightly increased, but not to the extent that warrants screening studies [33, 34].

Evaluation of the child with heterotaxy or situs inversus for malrotation is challenging. In children with the stomach on the right ("dextrogastria") the expected course of the duodenum is a mirror image to that seen in the child with situs solitus and no malrotation. Unfortunately, this has been termed "reversed rotation," which is ambiguous with the aforementioned "reversed rotation" seen in situs solitus [31]. To exclude malrotation in the setting of dextrogastria, the distal duodenum should extend to the right and upward, a mirror image of normal.

Malrotation may be seen in association with intestinal atresias and is perhaps contributory to development of atresia in some of these patients [17, 20]. In a large series by Vecchia et al. [35], $28 \%$ of infants with duodenal atresias had malrotation and $19 \%$ of infants with jejunoileal atresia had malrotation. The association 
between malrotation and jejunoileal atresia is stronger in children with abdominal wall defects, namely gastroschisis [35]. There is an increased incidence of malrotation in patients with cloacal extrophy and prune belly syndrome [36, 37]. Malrotation is seen in infants with megacystis-microcolon-intestinal hypoperistalsis syndrome (Berdon syndrome) [38] and is of increased incidence in children with intestinal neuronal dysplasia ("pseudo-obstruction") [39]. There are a few reported cases associated with Hirschsprung's disease [20]. Malrotation is weakly associated with some syndromes and chromosomal anomalies, the most notable of which is Down syndrome. The incidence of malrotation in children with Down syndrome is 45 times the incidence in children without Down syndrome [40].

\section{Imaging}

\section{Radiography}

The imaging work-up of a child with suspected malrotation begins with radiographs. For best results, two

Fig. 5 Changing bowel gas patterns with malrotation. a A film obtained shortly after birth (at 26 weeks gestation) shows the initial bolus of gas passing through proximal small bowel in the right abdomen, suggestive of malrotation. b A film obtained at 4 days of age shows a normal bowel gas pattern. c A film obtained at 27 days of age due to feeding intolerance shows dilated proximal duodenum (arrow) with a paucity of bowel gas distally. At surgery, a malrotation was found with the duodenum obstructed by overlying Ladd's bands; however, no volvulus was evident views of the abdomen are obtained - an anteroposterior supine view and either an anteroposterior upright view or a cross-table lateral view. Only rarely do the radiographs specifically suggest the diagnosis of malrotation. Rather, radiographs help to exclude other etiologies for the patient's symptoms and serve to guide further imaging. This is particularly true in the setting of a neonate with bilious vomiting - the bowel gas pattern will help differentiate distal from proximal obstruction.

The most common bowel gas pattern in the setting of malrotation is normal. In fact, in the setting of a normal bowel gas pattern in an infant with bilious vomiting, suspicion for malrotation should be heightened as opposed to other diagnoses. Berdon et al. [21] note:

"There is no more ominous finding in a suspected case of malrotation and volvulus than a "normal" plain film, since this may lead to delay and failure to pursue the diagnosis."

Findings suggesting an abnormal anatomical location of bowel include the presence of proximal small bowel on the right on early postnatal films following the first boluses of gas through the gastrointestinal tract (Fig. 5). Films in older children may occasionally show small bowel on the right and colon on the left, suggesting malrotation. Disproportionate dilatation of the duodenum with the "double bubble" appearance may be seen with severe duodenal obstruction due to volvulus or bands. With complete obstruction, this pattern is indistinguishable from duodenal atresia and other causes of congenital duodenal obstruction, and, in fact, these disorders, including malrotation, often occur concomitantly $[35,41]$. In an older infant or young child, the
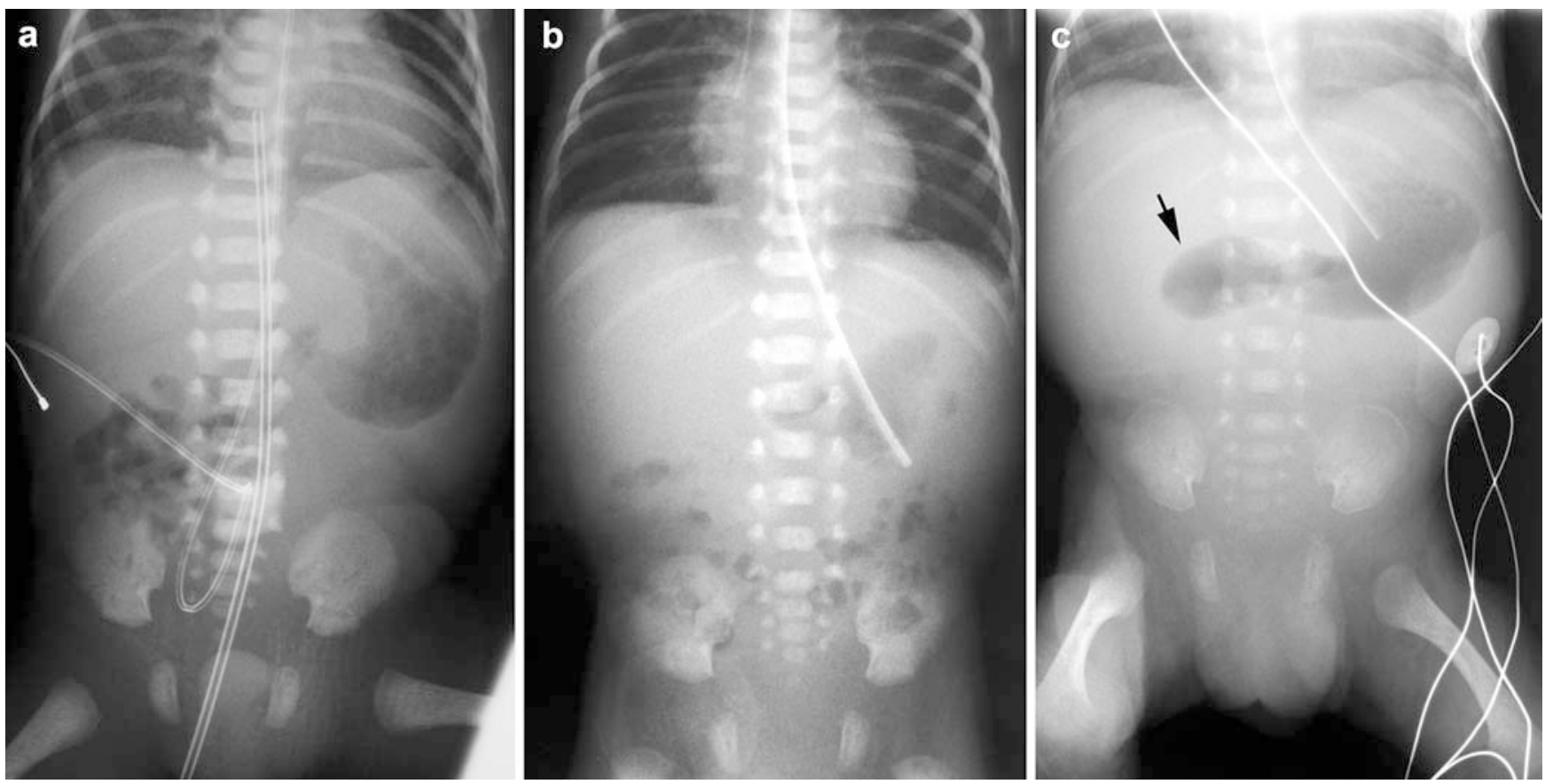
presence of a gas-filled, dilated duodenum and stomach with a paucity of bowel gas distally is very concerning for an acute duodenal obstruction, namely malrotation with volvulus or Ladd's bands obstructing the duodenum (Fig. 5c). A gasless abdomen may be due to malrotation with volvulus and obstruction [42-44]. A gasless abdomen associated with abdominal distension or tenderness may be a sign of strangulated midgut volvulus [43]. In the setting of volvulus, plain films may also show some mass effect in the mid-abdomen from the volvulized bowel. A "whirled" appearance of the bowel may suggest volvulus, but is rarely seen. With ischemia of the gut, separation of adjacent bowel loops, "tubular" appearing loops, fold thickening or "thumbprinting" may suggest abnormality (Fig. 6). These are grave signs. The presence of intermural gas or free intraperitoneal air at presentation is extremely rare, but also constitutes a grave prognostic sign. Diffuse gaseous distention of bowel from malrotation with volvulus is

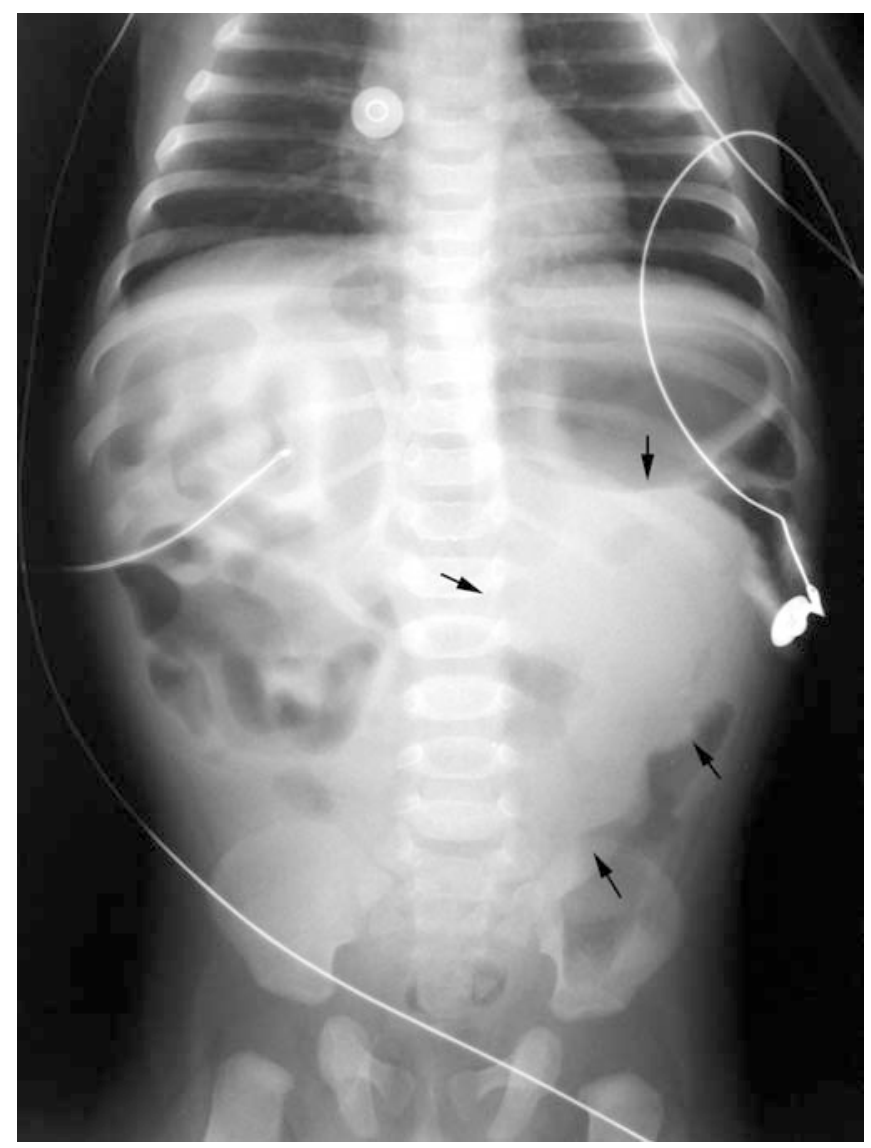

Fig. 6 Malrotation with volvulus: a 4-month-old male with obtundation and septic shock. "Tubular" appearing bowel loops are seen in the right upper quadrant. This proved to be ischemic small bowel. A mass-like opacity is seen in the mid-abdomen (arrows). At surgery, all but $2 \mathrm{~cm}$ of the intestinal tract was necrotic. The patient died distinctly uncommon and portends a poorer prognosis $[41,44]$. In a study by Frye et al. [44], a "low obstruction" pattern was highly correlated with gangrenous bowel. The authors hypothesized that vascular occlusion interferes with resorption of gas [43, 44].

\section{Upper GI}

An upper GI is the preferred modality for the radiologic diagnosis of malrotation and volvulus. The upper GI is usually preformed with barium, except in cases of a very sick infant or child in whom the presence of infarcted bowel and possible perforation are already suspected. In such instances, non-ionic water-soluble contrast medium should be utilized. A nasogastric tube aids in delivering and controlling the administration of contrast medium, but is not a necessity in all cases. Occasionally, advancement of a nasogastric tube into the duodenum may be of benefit in allowing fuller duodenal distention and thus better delineation of duodenal anatomy. Videotaping of fluoroscopy can be very helpful as transient findings can be difficult to capture on still images. A "last image hold" function is also helpful to capture still images of rapidly changing findings.

The normal duodenum has a C-shaped configuration consisting of four portions. The first portion of the duodenum, the bulb, and a short portion of post-bulbar duodenum are not fixed within the retroperitoneum, whereas the remainder of the second portion (descending), the third portion (transverse) and the fourth portion (ascending) are fixed within the retroperitoneum. Distally, the fourth portion of the duodenum extends cephalad and to the left. A slight flexure is seen where the duodenum turns into jejunum (the ligament of Treitz). This point should be to the left of the left spinal pedicle and should be near the level of the pylorus (Fig. 7). Since the second through the fourth portions of the duodenum are fixed in the retroperitoneum, they are posterior in location. As such, in the true lateral projection the fourth portion of the duodenum should project as posterior as the second portion of the duodenum (Fig. 8) [45, 46]. Slight rotation of the patient under fluoroscopic observation may be necessary to define this relationship. The importance of documenting the first bolus of contrast medium through the duodenum cannot be stressed enough. Once contrast medium has passed beyond the duodenum, loops of proximal jejunum may overlie the duodenum in both the anteroposterior and lateral projections, severely compromising assessment of the duodenal course. It is important not just to document the duodenum in the anteroposterior projection, but also in the lateral projection. This can be done by quickly rotating the patient to the lateral position once the duodenojejunal junction is reached. In patients in whom barium passage through the 


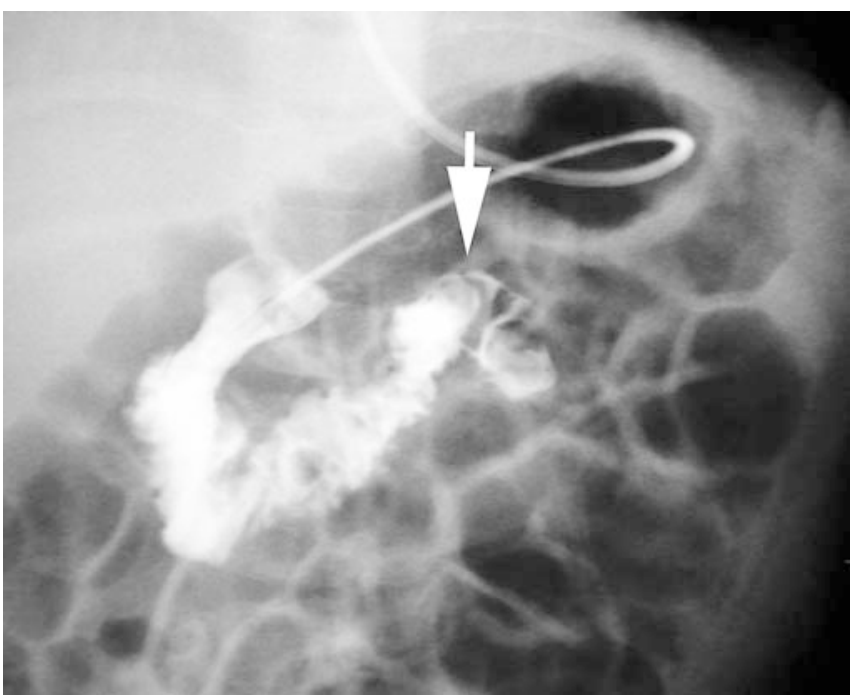

Fig. 7 Normal duodenal course: upper GI, anteroposterior projection-10-month-old boy. In this patient, evaluation of the duodenal course was aided by the presence an enteric tube crossing the pylorus. The duodenojejunal junction (arrow) is at the level of the pylorus and to the left of the left spinal pedicle

duodenum is slow, it may be helpful to place the patient right side down until contrast medium opacifies the first and second portions of the duodenum, then to turn the patient past the supine anteroposterior position toward the left side. With this maneuver, barium frequently passes into the distal duodenum and contrast is cleared from the gastric antrum. The patient is then quickly returned to the supine position and the anteroposterior

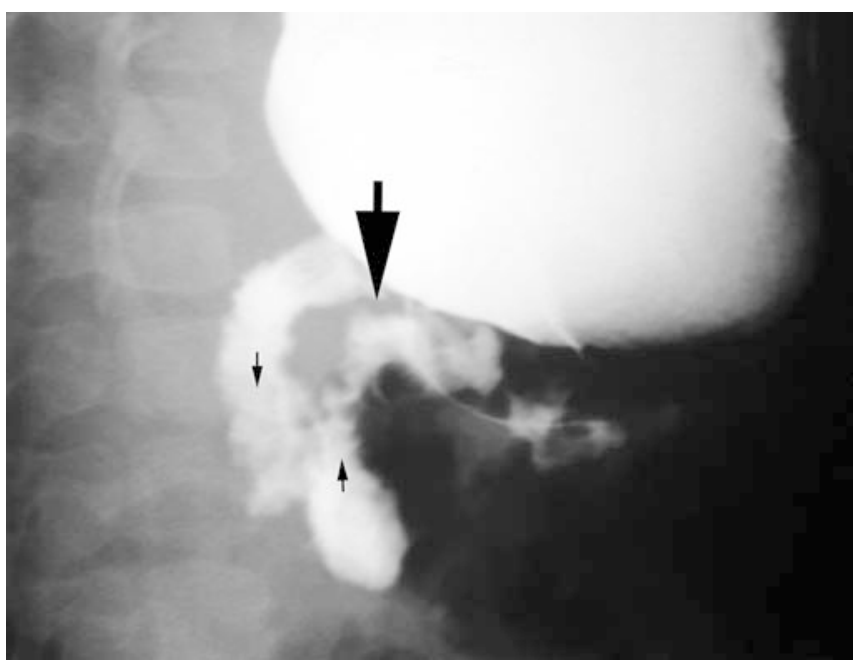

Fig. 8 Normal duodenal course: upper GI, lateral projection-14month-old girl. The second, third and fourth portions of the duodenum are posterior, within the retroperitoneum. Slight obliquity from true lateral allows visualization of both descending (small arrow down) and ascending (small arrow up) duodenum. The duodenojejunal junction (large arrow) is posterior in location duodenal course is well-delineated through a gas-filled gastric antrum. The patient is then quickly turned to the left to assess the position of the distal duodenum in the lateral projection. Alternatively, when barium reaches the distal duodenum, the duodenum is quickly assessed in the lateral projection; then the patient is quickly turned back supine to document the duodenojejunal junction in the anteroposterior projection.

The chief radiographic signs of malrotation on upper GI are: (1) abnormal position of the duodenojejunal junction, (2) spiral, "corkscrew" or Z-shaped course of the distal duodenum and proximal jejunum, and (3) location of the proximal jejunum in the right abdomen. With malrotation, the duodenal course is anomalous. The distal duodenum fails to extend as leftward and as cephalad as it should. The duodenojejunal junction is poorly defined. In most children with malrotation, the distal duodenum will take an anterior course on the lateral view rather than the normal posterior location [45]. Although rightward positioning of the proximal jejunum is a frequent finding in cases of malrotation this finding is not diagnostic of malrotation in the absence of other abnormality [47].

In classic volvulus, the distal duodenum and proximal jejunum follow a downward "corkscrew" course in the mid-abdomen (Fig. 9). The bowel lumen is narrowed with partial or complete obstruction. The duodenum proximal to the obstruction may be mildly dilated. At an obstruction, the bowel has a tapered or "beaked" appearance, usually extending downward. With complete obstruction, barium may not enter the volvulized loops to show a "corkscrew" (Fig. 10). Once the findings of volvulus are demonstrated, no further imaging is necessary. Prompt communication of the findings to the appropriate surgical team will expedite care.

In the newborn, and to a lesser extent in older children, malrotation not infrequently manifests as a proximal duodenal obstruction due to overlying bands. With a "double bubble" on plain films without distal bowel gas, it is unlikely that an upper GI will provide additional information [20]. Occasionally, if surgical management is to be delayed, an enema may be requested to exclude malrotation, as opposed to duodenal atresia; however, this is of limited value and the needs of the child are best assessed and met in the operating room, not the fluoroscopy suite. If the obstruction is not complete, what little contrast medium gets by the obstruction will document the duodenal course. Bands may also produce the Z-shaped configuration of the duodenum and proximal jejunum (Fig. 11). This is evident in both the anteroposterior and lateral projections [16]. The Z-shaped configuration may appear similar to the "corkscrew" of volvulus, but it does not indicate volvulus itself.

Factors other than malrotation may affect the duodenal course and render interpretation of an upper GI 
Fig. 9 Malrotation with volvulus: 1-day-old with bilious vomiting and abdominal distension. a Upper GI, anteroposterior projection. Barium opacifies a downward, narrow, "corkscrew" course of the distal duodenum and proximal jejunum (arrows). b Upper GI, lateral projection. The "corkscrew" pattern is again seen (white arrow). Note the anterior course of the distal duodenum (black arrow)
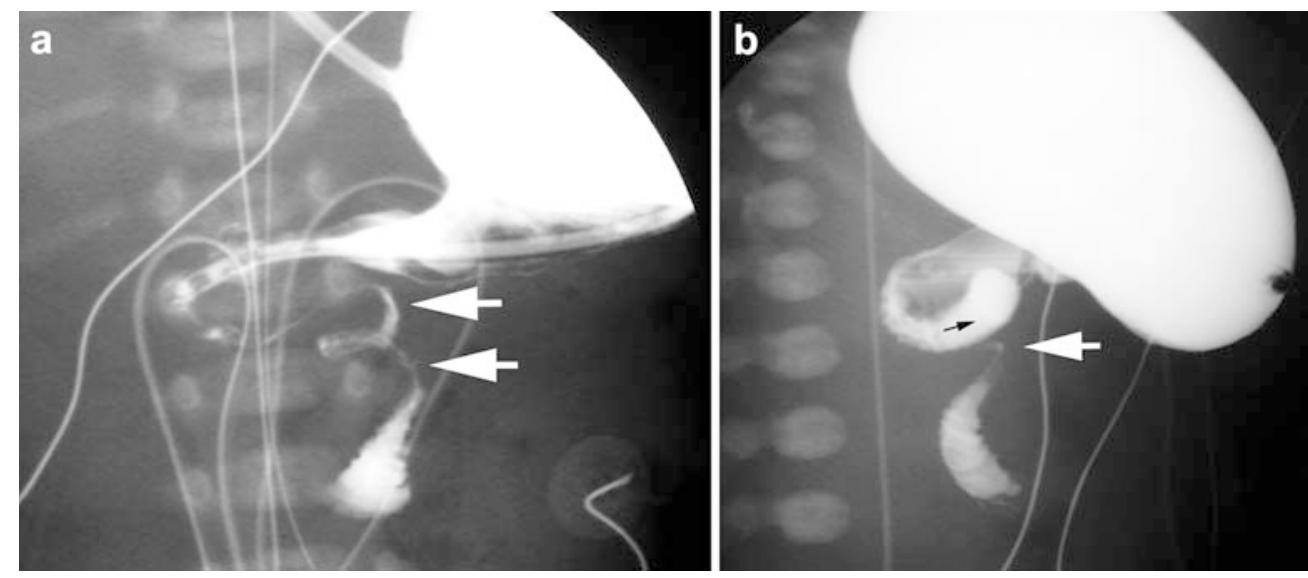

Fig. 10 Malrotation with volvulus: 5-week-old male with bilious vomiting. a Upper GI, anteroposterior projection. The distal duodenum does not ascend; rather it descends and tapers to a "beaked" obstruction (arrow). b Upper GI, lateral projection. Note the anterior course of the distal duodenum (arrow)
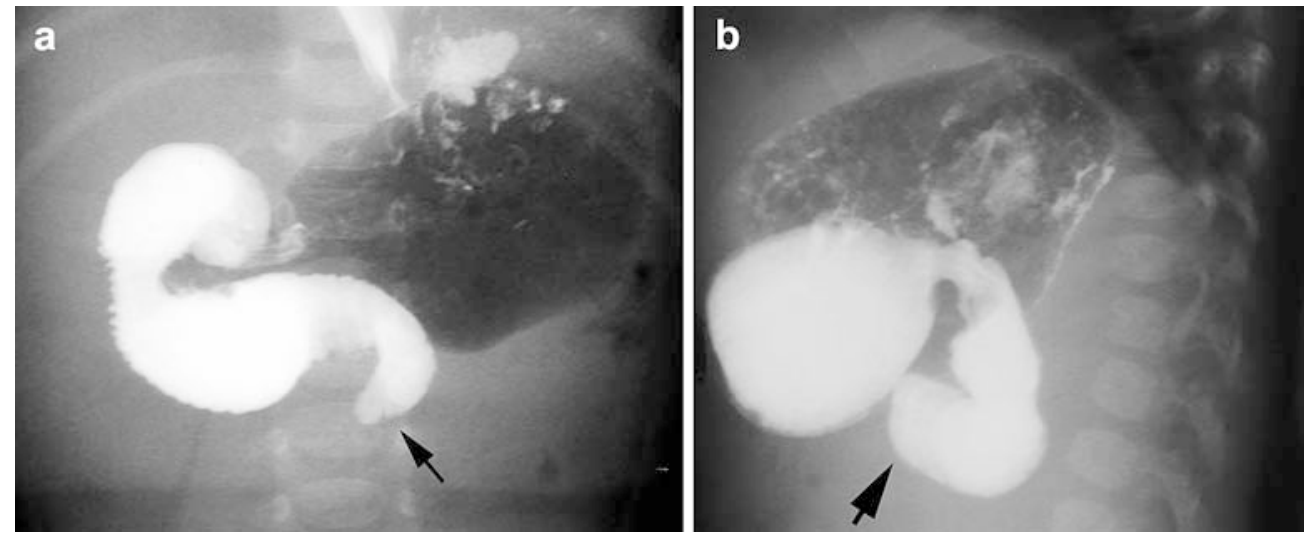

difficult. The duodenal course can be altered by preceding surgery, including duodenostomy for duodenal atresia and by liver transplant [48, 49]. In the latter,

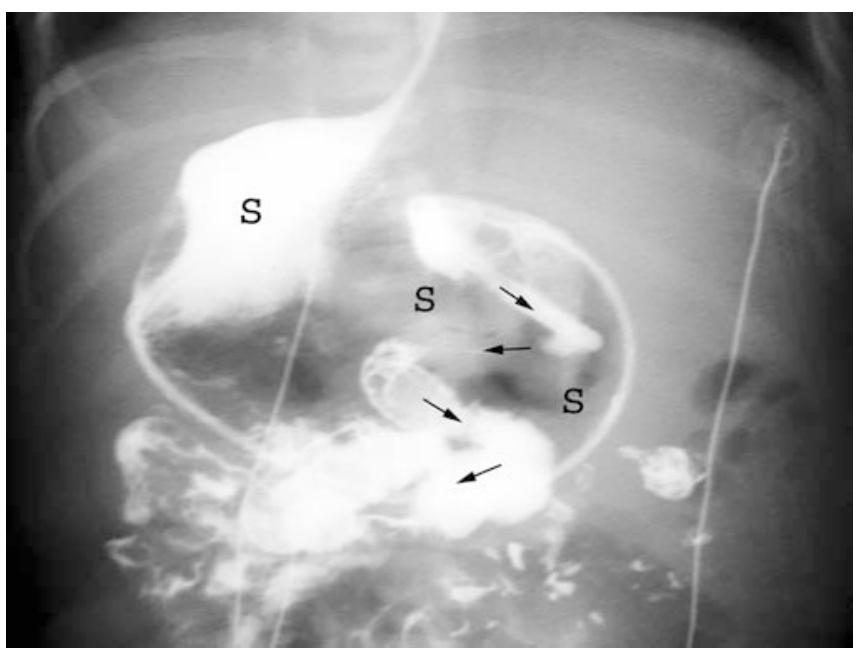

Fig. 11 Malrotation with Z-deformity of the duodenum: 3-day-old girl with polysplenia and feeding intolerance. The stomach $(S)$ is on the right. There is a downward, "zigzag" course (arrows) of the duodenum, accentuated by a feeding tube proximally. Malrotation with partially obstructing Ladd's bands was found at surgery deformity is greater with left lobe transplants, likely due to a mass effect from the graft or rightward displacement of the duodenum into the space vacated by the explanted liver [49]. Large masses in the upper abdomen will distort the duodenum. Marked gastric distention or chronic bowel dilatation may displace the distal duodenum medially and/or inferiorly (Fig. 12) [50]. Incomplete fixation in the infant allows for deformation of the duodenal course by an enteric tube [51]. Incomplete fixation of the duodenum may also be evident in premature infants as a mildly abnormal course [52]. Katz et al. [53] and Lim-Dunham et al. [54] have noted that the duodenojejunal junction can be manually displaced so as to appear abnormal in over two-thirds of infants. The normally fixated duodenum quickly returns to a normal configuration with release of compression, whereas the malfixated duodenum may remain abnormal in position after release of compression.

\section{Difficult cases}

In most patients, an upper GI will provide clear distinction of normalcy from malrotation. Unfortunately, and to the despair of radiologist and surgeon alike, not 


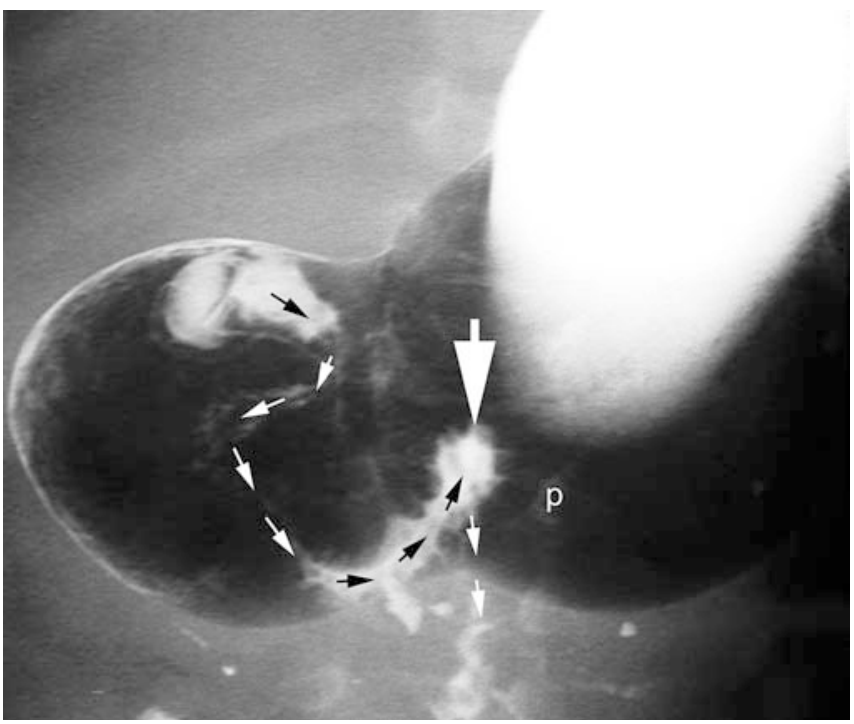

Fig. 12 Duodenal distortion due to marked gastric distension. Small arrows indicate the course of the duodenum and proximal jejunum. The large arrow indicates the duodenojejunal junction projecting near the midline ( $p$ left spinal pedicle). After gastric decompression, the duodenojejunal junction was at normal location. A normal location of the duodenojejunal junction had previously been confirmed at surgery performed for necrotizing enterocolitis

every upper GI study is definitively normal or definitively abnormal. As mentioned previously, malrotation represents a spectrum of abnormality. Moreover, within the realm of normalcy, there are variations which may occasionally be difficult to differentiate from malrotation. Katz et al. [53] identified nine criteria on upper GI useful in distinguishing normal from abnormal positioning of the duodenum and proximal jejunum. In their study, the presence of three or more abnormal criteria was indicative of malrotation, the presence of two criteria was indeterminate, and the presence of one criterion was likely normal, providing that the ileocecal position was normal [53]. Long et al. [47] tested the Katz criteria in a series of 23 "difficult diagnostic cases" -12 with false-positive upper GI and 11 with "subtle" rotational abnormality ("potential false-negatives"). All 12 false-positives were correctly categorized as normal variants by the Katz criteria; however, 5 of the 11 subtle cases received a score of 0 or 1 and would have been misdiagnosed by the Katz criteria and 3 of the cases received an indeterminate score of 2 [47]. According to Long et al. [47], false-positive cases resulted from failure to recognize normal variants: jejunum in the right upper quadrant as the sole abnormality, duodenojejunal junction over the left pedicle on the anteroposterior view, "duodenum inversum" (distal duodenum ascends on the right then crosses to the left where the duodenojejunal junction is fixed in a normal location) (Fig. 13), and "duodenum mobile" (a long postbulbar

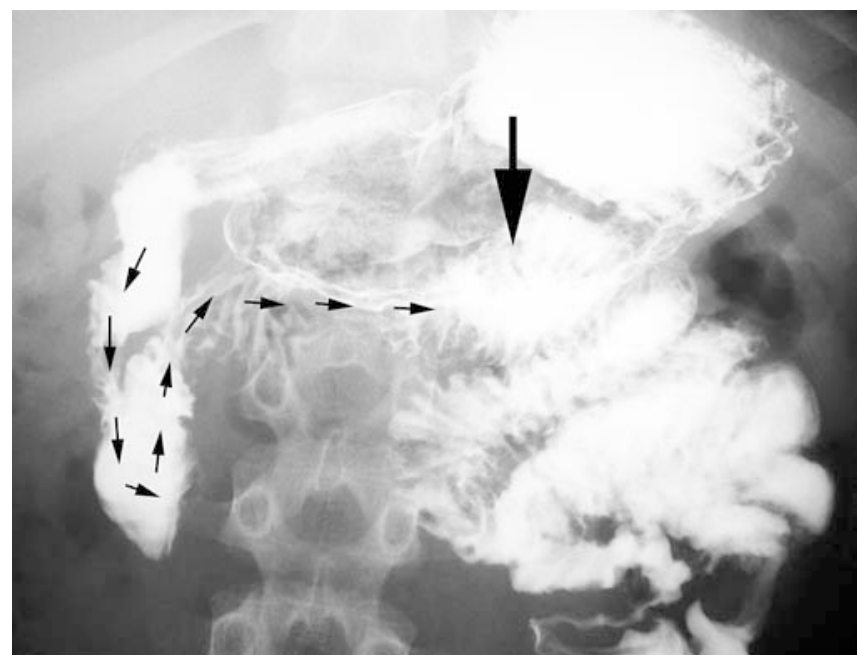

Fig. 13 Normal variant: duodenum inversum-11-year-old boy. The duodenum descends then ascends to the right of the spine, before crossing horizontally to the left (small arrows). The duodenojejunal junction is at a normal location (large arrow)

segment with undulation or redundancy due to lack of proximal duodenal fixation). Cecal mobility also contributed to false-positive interpretations [47]. Subtle signs of malrotation identified by them were unusual redundancy of the duodenum to the right of the spine and location of the duodenojejunal junction medial to the left spinal pedicle. Some redundancy of the duodenum is normal; however, too much redundancy is not normal. Angularity and kinking or formation of more than one loop in the course of the duodenal sweep are indicative of abnormal redundancy, and therefore, of malrotation (Fig. 14) [47].

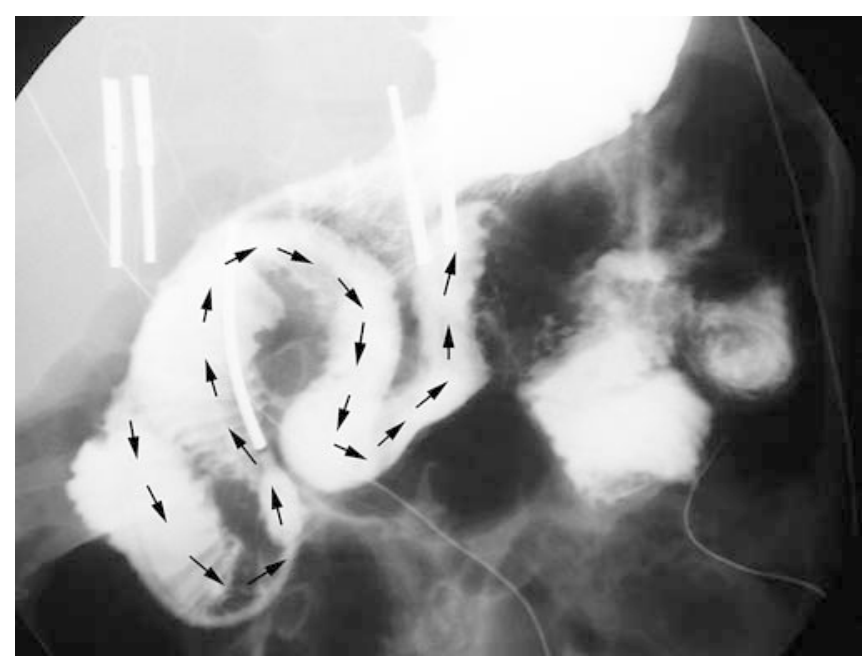

Fig. 14 Malrotation: 2-year-old girl with persistent non-bilious emesis. Arrows indicate the course of an excessively redundant duodenum. A feeding tube is present. Malrotation was confirmed at surgery 
The left spinal pedicle can be used to distinguish adequate right/left positioning of the duodenojejunal junction [47]. In terms of cephalocaudad position, various landmarks have been used to define normal: pylorus, duodenal bulb [20, 47], midway between lesser and greater curvatures of the stomach $[19,20]$. These landmarks are roughly equal in height and at approximately the L2 vertebral body. If the distal duodenum fails to ascend it is likely too low [53]. At our institution, we have found assessment of the duodenal course in the lateral projection to be a valuable adjunct in cases with equivocal findings in the anteroposterior projection (Fig. 15, also see Figs. 9 and 10).

Properly interpreted, a patient with volvulus will not have an equivocal upper GI. As such, patients with equivocal findings on upper GI may undergo further imaging without fear of immediate or impending compromise of the patient. Equivocal findings on upper GI warrant determination of cecal position. Demonstration of cecal position by following barium through the small bowel or performing an enema provides adjunctive information [10]. Theoretically, since contrast studies show position but only infer fixation, either an upper GI or a barium enema may be completely normal and false-negative $[10,55]$. There are reports of children with "normal" upper GI studies who had an obvious malrotation on follow-up studies [56]. The likelihood of malrotation when both studies are normal is extremely low $[10,46]$. If there is any diagnostic doubt or if malrotation is still suspected clinically despite normal findings on one study, it is necessary to evaluate the gastrointestinal tract from the other end [10]. Repetition of the upper GI, with increased attention to the duodenal course, perhaps through use of a nasoenteric tube with selective duodenal injection of contrast medium, may allow lessequivocal demonstration of duodenal anatomy. Very rarely, final diagnosis of an equivocal case still relies on surgery.

\section{Barium enema}

Historically, barium enema was performed for malrotation. The rationale for enema, as opposed to upper GI, was the common misperception that barium administered from above for the upper GI would be deleterious due to aspiration of vomitus or by changing an incomplete obstruction to a complete obstruction [21, 57]. In modern times, the enema has fallen out of favor for the diagnosis of malrotation for good reason. Approximately $20 \%$ of patients with malrotation will have a normally positioned cecum $[25,52,53]$. This is possible because the duodenojejunal and cecocolic segments do not rotate simultaneously [4]. Normal cecal position therefore does not exclude the diagnosis of malrotation [52]. Normal variations of cecal position and cecal mobility are common and form a continuum with abnormal variations of cecal position and excess cecal mobility from malfixation. Approximately $15 \%$ of patients have a mobile cecum $[4,6,46]$. Although many children with malrotation have a frankly abnormal cecal position, variations on normal and mildly abnormal may render interpretation difficult. Nevertheless, documentation of frankly abnormal or unequivocally abnormal cecal position in the setting of an equivocal upper GI may be of benefit. In fact, in a majority of patients with malrotation, an enema will be diagnostic for malrotation.

Obstruction of the colon itself due to malrotation is uncommon. Occasionally, with reflux of contrast medium into the small bowel, a "beaked" obstruction may indicate volvulus [58]. With the rare reversed rotation, the transverse colon may be obstructed as it passes posterior to the SMA [6]. Enemas performed primarily to evaluate for malrotation or for other reasons may show equivocal findings. In this situation, an upper GI should be performed to evaluate the duodenum.
Fig. 15 Malrotation: 1-day-old girl with polysplenia. a Upper GI, anteroposterior projection. The stomach $(S)$ is on the right. The proximal duodenal course is very redundant (arrows). Distally, the duodenum does not extend upward (large arrow). b Upper GI, lateral projection. Note the anterior course of the distal duodenum (arrow). A subsequent enema showed the colon to be entirely on the left. Malrotation was confirmed at surgery
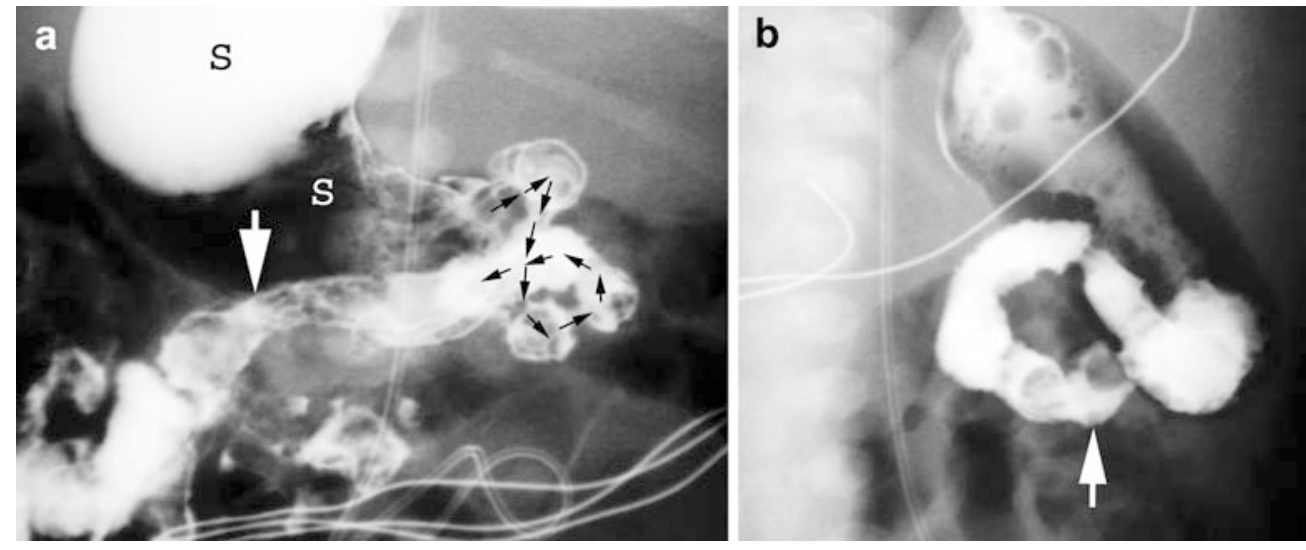


\section{Cross-sectional imaging}

US, CT and MRI are not preferred modalities for the diagnosis of malrotation. Nevertheless, these modalities, particularly US and CT, are used to image children with signs and symptoms of disease processes, which may present similarly to malrotation and malrotation with volvulus. Thus, it is important to recognize the findings of malrotation and volvulus on these studies when present.

Although uncommon in the neonate $(<30$ days of age), pyloric stenosis presents in the young infant with vomiting and thus may be confused clinically with malrotation. The nature of the vomitus usually distinguishes infants with pyloric stenosis (non-bilious emesis) from those with malrotation (bilious emesis). If a US examination is negative for pyloric stenosis, a quick look for findings of sonographic malrotation is warranted [59]. Inversion of the normal relationship of the SMA and vein has been described as a finding suggestive of malrotation [59-62]. Normally, the superior mesenteric vein is to the right of the artery. In malrotation, the vein is frequently to the left of the artery or rotates around the artery. The SMA is constant; however, superior mesenteric vein anatomy reflects the development and the anatomy of the bowel. Unfortunately, inversion of the normal relationship of the SMA and vein is neither highly specific nor highly sensitive, but when noted should warrant further evaluation for malrotation [60, $61,63]$. Some investigators have proposed using sonography to verify the entire duodenal course; however, this is too challenging for practical applicability [64].

Occasionally, sonography may demonstrate a dilated fluid-filled proximal duodenum proximal to an obstructing volvulus [61, 64, 65]. With volvulus, sonography may show a mass in the mid-abdomen with a "whirled" appearance of vasculature entering and within the volvulus - the "whirlpool" sign (Fig. 16) [61, $66,67]$. Other described Doppler US findings of volvulus include a truncated SMA [68], a solitary, hyperdynamic pulsating SMA [69] and a dilated distal superior mesenteric vein [61]. These findings are the sonographic correlates of the findings of malrotation with volvulus previously reported for angiography $[26,70]$.

Although unproven, CT is likely more sensitive than sonography in delineating the findings of malrotation. The diagnosis is not infrequently made on an incidental or unsuspected basis. CT may show malposition of bowel. Lack of the duodenum crossing inferior to the SMA from right to left should raise suspicion for malrotation. Abnormalities of situs or organ development may prompt a search for findings of malrotation. A small or hypoplastic pancreatic uncinate process is seen with malrotation, reflecting an interference of normal rotation of pancreatic primordia due to abnormal rotation

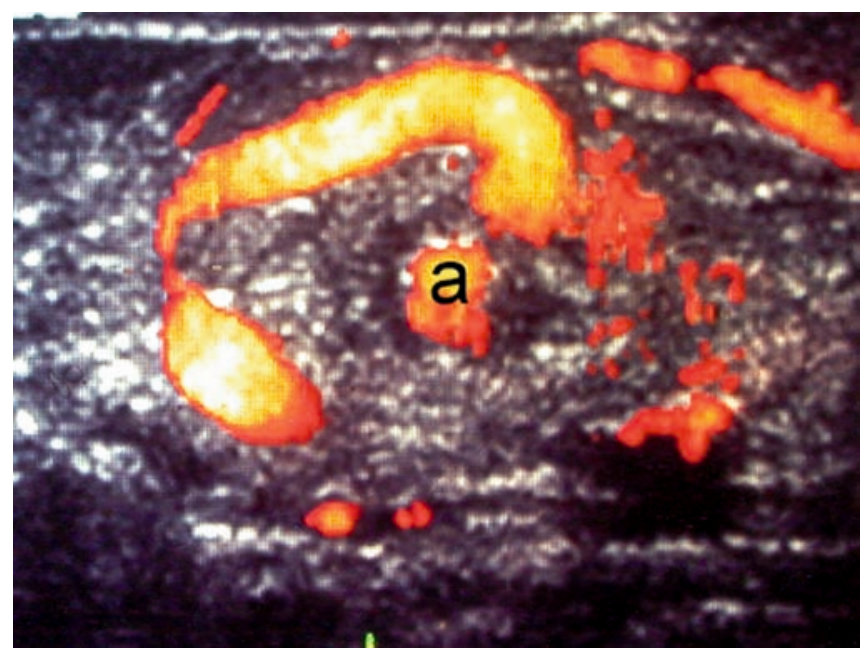

Fig. 16 Malrotation with volvulus: 5-day-old with bilious vomiting. An ultrasound was erroneously ordered to look for pyloric stenosis. Transverse power Doppler image shows the "whirlpool" sign indicative of volvulus ( $a$ SMA)

of bowel $[3,71]$. As with sonography, CT may show the anomalous orientation of the SMA and superior mesenteric vein (Fig. 17) or the "whirlpool" sign of volvulus $[63,72,73]$. In the setting of vascular compromise from malrotation, abnormal perfusion of bowel may be evident.

MR findings in malrotation are similar to those seen on CT, but are less manifest as patients with acute GI symptoms are not routinely imaged by MR and the spatial resolution of MR is less than that of CT. Like conventional angiography in the past, MR angiography may demonstrate abnormal vasculature suggestive of malrotation [74].

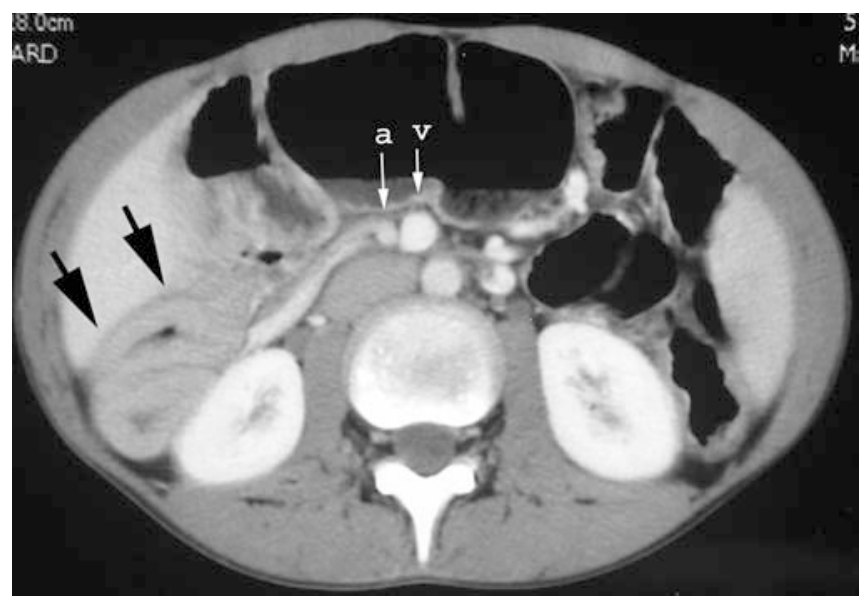

Fig. 17 Malrotation: 15-year-old boy with failure to thrive and malabsorption. Axial CT image shows the SMA $(a)$ to the right of the superior mesenteric vein $(v)$. Thick-walled small bowel loops are noted on the right (arrows) 


\section{Malrotation in the older child or adult}

Although increasingly uncommon with advancing age, presentation of malrotation with volvulus may occur at any age. Such patients may be previously asymptomatic or give a history of intermittent non-specific symptoms. The diagnosis of malrotation is often unsuspected, but findings are similar to those in younger children and should be recognized when present. Again, volvulus is a true surgical emergency. The symptomatic patient with malrotation warrants surgery; however, the approach to the older patient with an incidentally detected malrotation is controversial $[11,20,24,25,75,76]$. Many surgeons advocate a preventative Ladd's procedure in all patients with malrotation [24, 25, 76]. Others cautiously advocate conservative management, assuming that the diagnosis is incidental and the patient is truly asymptomatic [11]. Unfortunately, there are no guarantees that an asymptomatic patient with malrotation will remain asymptomatic and free from volvulus [24, 25, 76].

Complete nonrotation is not an infrequent incidental finding in adults. Most patients with nonrotation that are identified incidentally are asymptomatic and the risk for volvulus is low. Nevertheless, volvulus can occur [10, 77]. Nonrotation is less commonly associated with obstruction and volvulus than incomplete rotation because there is usually a broader mesenteric attachment with nonrotation [20]. Some authors have tried to stratify the risk for volvulus based on the radiographic anatomy [11]. The risk for volvulus is highest when the duodenojejunal junction is anomalous and the cecum is in the right upper quadrant or the left upper quadrant $[10,11,20]$. Cecal position in the right lower quadrant suggests a broader mesenteric attachment and a lower risk for volvulus. Unfortunately, only cecal fixation can be inferred from cecal position, and thus may be misleading in the case of a normally located but malfixated cecum [10]. If surgery is not pursued, the patient needs to be followed for development of symptoms and should be made knowledgeable of the potential consequences of volvulus so that evaluation and treatment are quickly sought and properly directed should symptoms arise.

\section{Surgery}

Historically, the preferred surgical approach to the patient with malrotation is the Ladd's procedure consisting of laparotomy with: (1) reduction of midgut volvulus, (2) division of peritoneal bands obstructing the duodenum, (3) placement of small and large bowel in a state of nonrotation, and (4) appendectomy [78, 79]. Today, most children with uncomplicated malrotation and some with malrotation with volvulus undergo a laparoscopic Ladd's procedure [79, 80]. Complicated cases with significant gut ischemia still demand an open approach. Today, the survival rate of children with malrotation with volvulus is high $(>80 \%)$; however, in spite of prompt diagnosis and prompt surgery, a significant minority of patients still die or suffer substantial morbidity due to loss of gut $[5,19,81]$. Factors associated with an increased mortality include: (1) younger age, (2) other clinical abnormalities, and (3) bowel necrosis [21, 81].

Care of the patient with malrotation does not cease with the Ladd's procedure. Recurrent volvulus is rare, but can occur [82]. Other postoperative complications may occur, including infection, obstruction from adhesions and stricture formation. Normalization of gut function occurs slowly in some children, prompting some to suggest that there is an underlying functional abnormality of gut innervation associated with or as a consequence of malrotation $[39,82]$.

\section{Conclusion}

In conclusion, diagnostic imaging of malrotation and malrotation with volvulus is challenging. The affected child often presents acutely and during off-hours. A well-performed upper GI is of paramount importance. Knowledge of the findings of malrotation, not just on upper GI, but also on other imaging modalities, is key to suspecting the diagnosis. Prompt diagnosis expedites prompt surgery and may be life-saving. The findings of malrotation may be subtle, but the consequences of missing the diagnosis may be grave.

"Discretion is the better part of valour." [William

Shakespeare, Henry IV, Part One, V, iv, 119]

\section{References}

1. Mall FP (1898) Development of the human intestine and its position in the adult. Bull Johns Hopkins Hosp 9091:197-208
2. Frazer JE, Robbins RH (1915) On the factors concerned in causing rotation of the intestine in man. J Anat Physiol 50:75-110
3. Dott NM (1923) Anomalies of intestinal rotation: their embryology and surgical aspects: with report of 5 cases. Br J Surg $24: 251-286$ 
4. Snyder WH Jr, Chaffin L (1954) Embryology and pathology of the intestinal tract: presentation of 40 cases of malrotation. Ann Surg 140:368-379

5. Skandalakis JE, Gray SW, Ricketts R, et al (1994) The small intestines. In: Skandalakis JE, Gray SW (eds) Embryology for surgeons, 2nd edn. Williams and Wilkins, Baltimore, pp 184-241

6. Jamieson D, Stringer DA (2000) Small bowel. In: Stringer DA, Babyn PS (eds) Pediatric gastrointestinal imaging and intervention, 2nd edn. BC Decker, Hamilton, pp 311-474

7. Estrada RL (1958) Anomalies of intestinal rotation and fixation. Charles $\mathrm{C}$. Thomas, Springfield

8. Haley JC, Peden JK (1943) The suspensory muscle of the duodenum. Am $\mathbf{J}$ Surg 59:546-550

9. Low FN, Hilderman WC (1940) A case of hyper-rotation of the colon. Anat Rec 77:27-30

10. Long FR, Kramer SS, Markowitz RI, et al (1996) Radiographic patterns of intestinal malrotation in children. Radiographics 16:547-556

11. Schey WL, Donaldson JS, Sty JR (1993) Malrotation of bowel: variable patterns with different surgical considerations. J Pediatr Surg 28:96-101

12. Waugh GE (1920) The morbid consequences of a mobile ascending colon, with a record of 180 operations. $\mathrm{Br} \mathbf{J}$ Surg 7:343-383

13. Brereton RJ, Taylor B, Hall CM (1986) Intussusception and intestinal malrotation in infants: Waugh's syndrome. Br J Surg 73:55-57

14. Zimmerman LM, Laufman H (1953) Intra-abdominal hernias due to developmental and rotational anomalies. Ann Surg 138:82-91

15. Donnelly LF, Rencken IO, deLorimier AA, et al (1996) Left paraduodenal hernia leading to ileal obstruction. Pediatr Radiol 26:534-536

16. Ablow RC, Hoffer FA, Seashore JH, et al (1983) Z-shaped duodenojejunal loop: sign of mesenteric fixation anomaly and congenital bands. AJR 141:461464

17. Miyakoshi K, Ishimoto $\mathrm{H}$, Tanigaki S, et al (2001) Prenatal diagnosis of midgut volvulus by sonography and magnetic resonance imaging. Am J Perinatol $18: 447-450$

18. Sabharwal G, Strouse PJ, Islam S, et al (2004) Congenital short-gut syndrome. Pediatr Radiol 34:424-427

19. Torres AM, Ziegler MM (1993) Malrotation of the intestine. World J Surg 17:326-331

20. Filston HC, Kirks DR (1981) Malrotation - the ubiquitous anomaly. J Pediatr Surg 16:614-620
21. Berdon WE, Baker DH, Bull S, et al (1970) Midgut malrotation and volvulus: which films are most helpful? Radiology 96:375-383

22. Godbole P, Stringer MD (2002) Biliou vomiting in the newborn: how often is it pathologic? J Pediatr Surg 37:909-911

23. Bonadio WA, Clarkson T, Naus J (1991) The clinical features of children with malrotation of the intestine. Pediatr Emerg Care 7:348-349

24. Spigland N, Brandt ML, Yazbeck S (1990) Malrotation presenting beyond the neonatal period. J Pediatr Surg 25:1139-1142

25. Prasil P, Flageole H, Shaw KS, et al (2000) Should malrotation in children be treated differently according to age? J Pediatr Surg 35:756-758

26. Howell CG, Vozza F, Shaw S, et al (1982) Malrotation, malnutrition, and ischemic bowel disease. J Pediatr Surg $17: 469-473$

27. Mori H, Hayashi K, Futagawa S, et al (1987) Vascular compromise in chronic volvulus with midgut malrotation. Pediatr Radiol 17:277-281

28. Levin TL, Liebling MS, Ruzal-Shapiro C, et al (1995) Midgut malfixation in patients with congenital diaphragmatic hernia: what is the risk of midgut volvulus? Pediatr Radiol 25:259-261

29. Teele RL, Pease PW, Rowley RS (1998) Malrotation in newborns following antenatal diagnosis of intra-abdominal cyst. Pediatr Radiol 28:717-721

30. Applegate KE, Goske MJ, Pierce G, et al (1999) Situs revisited: imaging of the heterotaxy syndrome. Radiographics 19:837-852

31. Ditchfield MR, Hutson JM (1998) Intestinal rotational abnormalities in polysplenia and asplenia syndromes. Pediatr Radiol 28:303-306

32. Moller JH, Amplatz K, Wolfson J (1971) Malrotation of the bowel in patients with congenital heart disease associated with splenic anomalies. Radiology 99:393-398

33. Fulcher AS, Turner MA (2002) Abdominal manifestations of situs anomalies in adults. Radiographics 22:1439-1456

34. Chang J, Brueckner M, Touloukian RJ (1993) Intestinal rotation and fixation abnormalities in heterotaxia: early detection and management. J Pediatr Surg 28:1281-1285

35. Vecchia LKD, Grosfeld JL, West KW, et al (1998) Intestinal atresia and stenosis. Arch Surg 133:490-497

36. Wright JR Jr, Barth RF, Neff JC, et a (1986) Gastrointestinal malformations associated with prune belly syndrome: three cases and a review of the literature. Pediatr Pathol 5:421-448
37. Meglin AJ, Balotin RJ, Jelinek JS, et al (1990) Cloacal exstrophy: radiologic findings in 13 patients. AJR 155:12671272

38. Berdon WE, Baker DH, Blanc WA, et al (1976) Megacystis-microcolonintestinal hypoperistalsis syndrome: a new cause of intestinal obstruction in the newborn. Report of radiologic findings in five newborn girls. AJR 126:957-964

39. Devane SP, Coombes R, Smith VV, et al (1992) Persistent gastrointestinal symptoms after correction of malrotation. Arch Dis Child 67:218-221

40. Torfs CP, Christianson RE (1998) Anomalies in Down syndrome individuals in a large population-based registry. Am J Med Genet 77:431-438

41. Houston CS, Wittenborg MH (1965) Roentgen evaluation of anomalies of rotation and fixation of the bowel in children. Radiology 84:1-16

42. LoPresti JM, Majd M, Randolph JG (1972) The "airless" abdomen in the newborn infant. South Med J 65:309312

43. Kassner EG, Kottmeier PK (1975) Absence and retention of small bowel gas in infants with midgut volvulus: mechanisms and significance. Pediatr Radiol 4:28-30

44. Frye TR, Mah CL, Schiller M (1972) Roentgenographic evidence of gangrenous bowel in midgut volvulus with observations in experimental volvulus. Am J Roentgenol Radium Ther Nucl Med 114:394-401

45. Koplewitz BZ, Daneman A (1999) The lateral view: a useful adjunct in the diagnosis of malrotation. Pediatr Radiol 29:144-145

46. Beasley SW, De Campo JF (1987) Pitfalls in the radiological diagnosis of malrotation. Australas Radiol 33:376383

47. Long FR, Kramer SS, Markowitz RI, et al (1996) Intestinal malrotation in children: tutorial on radiographic diagnosis in difficult cases. Radiology 198:775-780

48. Zerin JM, Polley TZ Jr (1994) Malrotation in patients with duodenal atresia: a true association or an expected finding on postoperative upper gastrointestinal barium study? Pediatr Radiol 24:170 172

49. Benya EC, Ben-Ami TE, Whitington PF, et al (1998) Duodenum and duodenal-jejunal junction in children: position and appearance after liver transplantation. Radiology 207:233-236

50. Taylor GA, Teele RL (1985) Chronic intestinal obstruction mimicking malrotation in children. Pediatr Radiol 15:392-394 
51. Merten DF, Mumford L, Filston HC, et al (1980) Radiological observations during transpyloric tube feeding in infants of low birth weight. Radiology 136:67-75

52. Slovis TL, Klein MD, Watts FB (1980) Incomplete rotation of the intestine with a normal cecal position. Surgery $87: 325-330$

53. Katz ME, Siegel MJ, Shackelford GD, et al (1987) The position and mobility of the duodenum in children. AJR 148:947-951

54. Lim-Dunham JE, Ben-Ami T, Yousefzadeh DK (1999) Manual epigastric compression during upper gastrointestinal examination of neonates: value in diagnosis of intestinal malrotation and volvulus. AJR 173:979-983

55. Stringer DA (1996) Invited commentary. Radiographics 16:556-558

56. Blumberg K (1997) Intestinal malrotation (letter to the editor). Radiology 202:584

57. Silverman FN, Caffey J (1949) Congenital obstructions of the alimentary tract in infants and children: errors of rotation of the midgut. Radiology 53:781-787

58. Siegel MJ, Shackelford GD, McAlister WH (1980) Small bowel volvulus in children: its appearance on the barium enema examination. Pediatr Radiol 10:91-93

59. Weinberger E, Winters WD, Liddell RM, et al (1992) Sonographic diagnosis of intestinal malrotation in infants: importance of the relative positions of the superior mesenteric vein and artery. AJR 159:825-828

60. Zerin JM, DiPietro MA (1992) Superior mesenteric vascular anatomy at US in patients with surgically proved malrotation of the midgut. Radiology 183:693-694
61. Chao H-C, Kong M-S, Chen J-Y, et al (2000) Sonographic features related to volvulus in neonatal intestinal malrotation. J Ultrasound Med 19:371-376

62. Dufour D, Delaet MH, Dassonville M, et al (1992) Midgut malrotation, the reliability of sonographic diagnosis. Pediatr Radiol 22:21-23

63. Zerin JM, DiPietro MA (1991) Mesenteric vascular anatomy at CT: normal and abnormal appearances. Radiology 179:739-742

64. Cohen HL, Haller JO, Mestel AL, et al (1987) Neonatal duodenum: fluid-aided US examination. Radiology 164:805809

65. Leonidas JC, Magid N, Soberman N, et al (1991) Midgut volvulus in infants: diagnosis with US. Radiology 179:491493

66. Shimanuki Y, Aihara T, Takano H, et al (1996) Clockwise whirlpool sign at color doppler US: an objective and definite sign of midgut volvulus. Radiology 199:261-264

67. Pracros JP, Sann L, Genin G, et al (1992) Ultrasound diagnosis of midgut volvulus: the "whirlpool" sign. Pediatr Radiol 22:18-20

68. Sze RW, Guillerman RP, Krauter D, et al (2002) A possible new ancillary sign for diagnosing midgut volvulus: the truncated superior mesenteric artery. $\mathbf{J}$ Ultrasound Med 21:477-480

69. Smet M-H, Marchal G, Ceulemans R, et al (1991) The solitary hyperdynamic pulsating superior mesenteric artery: an additional dynamic sonographic feature of midgut volvulus. Pediatr Radiol 21:156-157

70. Buranasiri SI, Baum S, Nasbaum M, et al (1973) The angiographic diagnosis of mid-gut malrotation with volvulus in adults. Radiology 109:555-556

71. Inoue Y, Nakamura H (1997) Aplasia or hypoplasia of the pancreatic uncinate process: comparison in patients with and patients without intestinal nonrotation. Radiology 205:531-533
72. Nichols DM, Li DK (1983) Superior mesenteric vein rotation: a CT sign of midgut malrotation. AJR 141:707-708

73. Bernstein SM, Russ PD (1998) Midgut volvulus: a rare cause of acute abdomen in an adult patient. AJR 171:639-641

74. Yamamoto R, Nagao S, Hashiguchi K, et al (2003) An adult case of midgut volvulus with malrotation diagnosed by MR angiography. Nippon Shokakibyo Gakkai Zasshi 100:1307-1311

75. Dilley AV, Pereira J, Shi ECP, et al (2000) The radiologist says malrotation: does the surgeon operate? Pediatr Surg Int 16:45-49

76. Powell DM, Othersen B, Smith CD (1989) Malrotation of the intestines in children: the effect of age on presentation and therapy. J Pediatr Surg 24:777780

77. Simpson AJ, Leonida JC, Krasna IH, et al (1972) Roentgen diagnosis of midgut malrotation: value of upper gastrointestinal radiographic study. $\mathbf{J}$ Pediatr Surg 7:243-252

78. Ladd WE (1936) Surgical diseases of the alimentary tract in infants. $\mathbf{N}$ Engl $\mathbf{J}$ Med 215:705-708

79. Mazziotti MV, Strasberg SM, Langer JC (1997) Intestinal rotation abnormalities without volvulus: the role of laparoscopy. J Am Coll Surg 185:172176

80. Bass KD, Rothenberg SS, Chang JHT (1998) Laparoscopic Ladd's procedure in infants with malrotation. J Pediatr Surg 33:279-281

81. Messineo A, MacMillan JH, Palder SB, et al (1992) Clinical factors affecting mortality in children with malrotation of the intestine. J Pediatr Surg 27:13431345

82. Feitz R, Vos A (1997) Malrotation: the postoperative period. J Pediatr Surg 32:1322-1324 